\title{
Low platelet counts predict mortality in severe community acquired pneumonia in children under 5 years of age: A hospital based observational study
}

\author{
Anubha Jain*, Nidhi Awasthi, Shally Awasthi \\ Department of Pediatrics, King George's Medical University, Lucknow, Uttar Pradesh, India
}

\section{A R T I C L E I N F O}

\section{Keywords:}

Children

Community acquired pneumonia

Platelet counts Mortality

\begin{abstract}
A B S T R A C T
Objective: To assess the association of clinical and common laboratory parameters (haemoglobin, leukocyte and platelet count) with mortality in hospitalized cases with CAP.

Methods: A prospective observational study, conducted in a tertiary health institute in Lucknow, Northern India. Included were children between 2 to 59 months admitted with WHO defined severe pneumonia. Excluded were those illness of $\geq 14$ days, prior hospitalization for same illness and who were on anti-tubercular therapy and having fast breathing due to congenital heart disease.

Results: From August 2014 to July 2015, of 196 patients admitted, 152 were included of which 11 (7.2\%) expired. On unconditional logistic regression analysis, controlling for abnormal sensorium, cyanosis, and severe acute malnutrition, platelet count $<0.7 \mathrm{lac} / \mathrm{mm} 3$ on admission predicted mortality (adjusted OR $=10.7$, $\mathrm{p}<0.03$ ) and had good specificity (95.4\%) but low sensitivity (45.5\%).

Conclusion: Since low platelet count is an independent predictor of mortality in children with severe CAP, it must be estimated routinely in all cases.
\end{abstract}

\section{Introduction}

Community Acquired Pneumonia (CAP) is the leading cause of mortality in children under five years of age. In 2015, WHO estimated that 920136 children died of CAP, which accounted for $16 \%$ of total mortality in children under five years ${ }^{1}$. Revised guidelines of WHO classified pneumonia into two categories a) pneumonia which can be treated at home with the oral dose of amoxicillin and, b) severe pneumonia is treated with injectable antibiotics ${ }^{2}$.

Reported predictors of mortality in CAP are altered sensorium, abnormal total leukocyte count, pallor, malnutrition in young age and incomplete immunization ${ }^{3-5}$. Some studies have also reported low platelet count as a predictor of mortality in children admitted in Intensive care unit (ICU) as well as in adults with sepsis $6,8,9,106,8-10$. Children with severe CAP may also have concomitant sepsis which places them at increased risk of mortality ${ }^{11}$.There is a lack of studies looking into association of low platelet counts with mortality in children with CAP with sepsis.

Therefore the objective of current study was to assess the association of low platelet count with mortality in cases hospitalized with CAP.

\section{Materials and methods}

This was a prospective observational study, conducted in the Department of Pediatrics, King George's Medical University, a tertiary care teaching institute, in Lucknow, India after obtaining ethical approval from the institutional ethics committee.

Included were children aged between 2 months to 59 months admitted with WHO defined CAP ${ }^{1}$ after obtaining written informed parental consent. Excluded were children with illness of more than 14 days, prior hospitalization for same illness and who were already taking anti-tubercular therapy and who had fast breathing due to congenital heart disease. WHO criteria were used to define CAP, that is a) fast breathing and/or chest indrawing that is as pneumonia b) pneumonia with any danger sign (e.g. altered consciousness, convulsion, inability to feed) as severe pneumonia. On the basis of anthropometric measurements children were classified as having no malnutrition, moderate malnutrition, (weight for height $(\mathrm{WH}), \mathrm{Z}$ score between $<-1 \mathrm{SD}$ to $<-2 \mathrm{SD}$ ), severe acute malnutrition (WH, $\mathrm{Z}$ score $<-3 \mathrm{SD}$ ). Severe acute malnutrition (SAM) was defined as WH, Z score $<-3 \mathrm{SD}^{12}$. Severe anaemia was defined as hemoglobin level $<7 \mathrm{gm} / \mathrm{dl}^{13}$.

Complications of the CAP were one or more of the following;

\footnotetext{
* Corresponding author.

E-mail addresses: anubhajain15@gmail.com (A. Jain), awasthivn75@gmail.com (N. Awasthi), shally07@gmail.com (S. Awasthi).
} 
pyogenic meningitis, blood culture positive bacteremia, pleural effusion, acute respiratory distress syndrome, pyopericardium or septic arthritis.

Routine haematological parameters were analyzed by automated cell count analyzer (Swelab cell counter) based on the principle of optical scattering.

\section{Data collection}

All the data including demographic (e.g. age, sex, immunization history), anthropometric (e.g. weight, height, mid upper arm circumference) and clinical variables (sensorium, pallor, cyanosis, nasal flaring, breath sound, meningeal signs etc. on admission), laboratory and outcome variables such as discharged or expired and duration of hospital stay, were recorded in predesigned questionnaire. Clinical features such as symptoms of illness with their duration, respiratory rate, heart rate, oxygen saturation by pulse oxymetry, were abstracted from the hospital records. Complications on admission or during hospital stay were noted. Patients were followed up till final outcome (discharge or death).

\section{Anthropometric measurements}

Weight (in kg) in children, was measured by electronic weighing machine. Height (in $\mathrm{cm}$ ) in the children $\geq 2$ years of age was recorded by stadiometer while infantometer was used to measure the length in younger children. Mid arm circumference (in $\mathrm{cm}$ ) was measured with non-stretchable measuring tape. All the digits were corrected to one decimal unit. For sample size calculation we assumed $5 \%$ mortality in hospitalized cases of CAP. To detect this proportion with precision of $5 \%$ and alpha level of 0.05 . We needed to enrol a minimum of 73 cases. However we planned to recruit all eligible cases admitted in 1 year (Aug 2014-Jul 2015).

\section{Statistical analysis}

All the data were entered in MS Excel and analyzed in SPSS software (version 17.0). Univariate analysis was performed to study the frequency distribution of the variables. Variables were compared between those who expired or were discharged. Statistical differences between

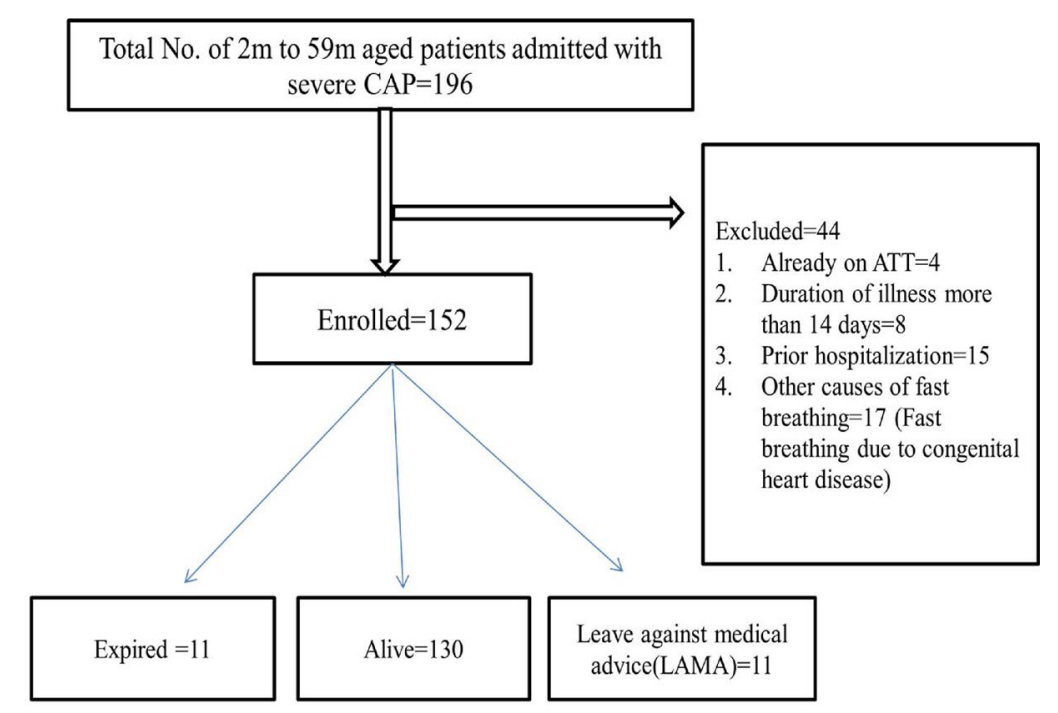

CAP, community acquired pneumonia ; ATT, anti tubercular treatment

Fig. 1. Total number of patients, aged $2 \mathrm{~m}$ to 59 months admitted with severe CAP.

Table 1

Clinical variables and laboratory parameters associated with mortality in hospitalized severe Community acquired pneumonia.

\begin{tabular}{|c|c|c|c|c|c|c|}
\hline \multirow[t]{2}{*}{ S. No. } & \multirow[t]{2}{*}{ Clinical Variables } & \multirow[t]{2}{*}{$\mathrm{N}$} & \multicolumn{2}{|l|}{ Outcome } & \multirow[t]{2}{*}{ OR $(95 \% \mathrm{CI})$} & \multirow[t]{2}{*}{ P Value } \\
\hline & & & $\operatorname{Expired}(\mathrm{n}=11)$ & Alive $(\mathrm{n}=130)$ & & \\
\hline 1 & Abnormal sensorium & 33 & $9(82)$ & $24(18.5)$ & $19.87(4.03-97.94)$ & $<0.001$ \\
\hline 2 & Cyanosis & 9 & $5(45)$ & $4(3)$ & $26.25(5.57-123.55)$ & $<0.0001$ \\
\hline 3 & $\begin{array}{l}\text { Severe acute } \\
\text { malnutrition }\end{array}$ & 24 & $6(54.5)$ & 18(13.8) & $7.46(2.06-27.04)$ & 0.002 \\
\hline 4 & Any Complications & 29 & $5(45)$ & $24(18.5)$ & $3.68(1.03-13.06)$ & 0.043 \\
\hline
\end{tabular}

Laboratory parameters

\begin{tabular}{|c|c|c|c|c|}
\hline S. No. & Parameter & Expired(n $=11)$ Mean $\pm S D$, (median) & Alive $(\mathrm{n}=130)$ Mean \pm SD, (median) & $P$ value \\
\hline 1 & Hemoglobin(gm/dl) & $8.61 \pm 1.11(8.2)$ & $9.74 \pm 1.57(9.4)$ & 0.021 \\
\hline 2 & Total Leucocyte count $(/ \mathrm{mm} 3)$ & $19,727 \pm 7550(22,000)$ & $16,255 \pm 7017(14,530)$ & 0.051 \\
\hline \multirow[t]{2}{*}{3} & Platelet count (lac/mm3) & $0.82 \pm 0.47(0.7)$ & $2.98 \pm 1.30(2.85)$ & \\
\hline & & & & $<0.0001$ \\
\hline
\end{tabular}

Complications: Pyogenic meningitis-5(17.2\%); Blood culture positive-5(17.2\%); Pleural effusion-19(65.6\%). 
Table 2

Predictors of mortality in patient admitted with community acquired pneumonia.

\begin{tabular}{|c|c|c|c|c|c|}
\hline \multirow[t]{2}{*}{ S. No. } & \multirow[t]{2}{*}{ Predictors } & \multicolumn{2}{|c|}{ Outcome } & \multirow[t]{2}{*}{ Adjusted OR (95\% CI) } & \multirow[t]{2}{*}{ P Value } \\
\hline & & $\begin{array}{c}\text { Expired } \\
(\mathrm{n}=11)(\%)\end{array}$ & $\begin{array}{c}\text { Alive } \\
(\mathrm{n}=130)(\%)\end{array}$ & & \\
\hline 1. & Male gender $(\mathrm{n}=92)$ & $8(72)$ & $76(58)$ & $0.87(0.10-7.49)$ & 0.90 \\
\hline 2. & Abnormal Sensorium & $2(18)$ & $105(81)$ & $21.78(2.004-236.74)$ & 0.01 \\
\hline 3 & Cyanosis & $5(45)$ & $4(3)$ & $10.22(1.18-88.14)$ & 0.03 \\
\hline 4. & Severe acute malnutrition $(\mathrm{N}=24)$ & $6(54.5)$ & $18(14)$ & $8.02(1.20-53.39)$ & 0.031 \\
\hline 5. & Severe anemia & $1(9)$ & $2(1.5)$ & $8.62(0.01-9.34)$ & 0.54 \\
\hline 6. & Platelet $\operatorname{count}(<0.7$ lac $/ \mathrm{mm} 3)(\mathrm{n}=11)$ & $5(45)$ & $6(4)$ & $10.71(1.31-78.69)$ & 0.03 \\
\hline
\end{tabular}

continuous variables were analyzed by Student ' $\mathrm{t}$ ' and categorical variables by Chi Square test to assess the association of categorical variables with mortality Crude odd's ratio with its $95 \% \mathrm{CI}$ was calculated. A p value of $<0.05$ was taken as statistically significant using a 2-tailed distribution.

In unconditional logistic regression analysis all categorical variables which had univariate association with mortality with a $\mathrm{p}$ value of $<0.1$ and were clinically meaningful were used as potential predictors of mortality.

We analyzed sensitivity and specificity of 10th of platelet count among cases recruited in the study for predicting mortality.

\section{Results}

A total of 196 patients of pneumonia were admitted from August 2014 to July 2015.We recruited 152 patients of CAP according to inclusion criteria (Fig. 1). Among those, 84 (55.3\%) were $\leq 12$ months of age and $68(44.7 \%)$ were $>12$ month of age, $92(60.5 \%)$ were male, 96 (63.2\%) were completely immunized and $18(11.8 \%)$ were partially immunized. Mean age of the patients was 19.51( \pm 18.27) months. Mean weight of male and female cases was $7.51 \pm 3.06 \mathrm{~kg}$ and $7.87 \pm 3.22 \mathrm{~kg}$, respectively and mean height of $70.90 \pm 16.48 \mathrm{~cm}$ and $70.94 \pm 14.95 \mathrm{~cm}$, respectively. Pallor was found in $41(27.0 \%)$ and added sounds (crepts/rocnchi) were found in $94.5 \%$. Salient clinical and laboratory findings have been compared between those who expired versus who were discharged or alive (Table 1 ).

Mean platelet count was $2.76( \pm 1.42) \mathrm{lac} / \mathrm{mm} 3$ and was statistically significantly lower in those who expired (Table 1 ). For 10th percentile cut off was $<0.7 \mathrm{lac} / \mathrm{mm} 3$ which had sensitivity of $45.5 \%$ and specificity of $95.4 \%$ to predict mortality of platelet count.

In the multivariate unconditional logistic regression analysis, controlling for danger signs such as altered sensorium, cyanosis, and SAM, platelet count of $<0.7 \mathrm{lac} / \mathrm{mm} 3$ was found as independent predictor of mortality in patients hospitalized with CAP (Table 2).

Complications such as pyogenic meningitis and blood culture positive were found each in 5 cases and there was presence of at least one or more complication was higher in the individuals who expired or discharged with any complication.

\section{Discussion}

The current study was conducted to determine the predictors of mortality in the patients aged 2 month to 59 months hospitalised with CAP and the association of low platelet count with mortality.

We found severe acute malnutrition, cyanosis and altered level of consciousness as independent predictors of mortality as reported by others ${ }^{3,4}$.

We also found platelet counts on admission to be significantly lower among those who expired. Platelet count $<0.7$ lac $/ \mathrm{mm} 3$ (10th percentile for all recruited cases) was an independent predictor of mortality along with SAM, abnormal sensorium and cyanosis.

The current study did not find any association of total leukocyte count and pallor with mortality as reported by others ${ }^{3,4}$. The reason for this variation may be due to different prevalence of anemia/pallor in the study population.

Other studies ${ }^{7-9}$ reported the association of low platelet count with sepsis in children. Similarly, in adults, it has also been reported that low platelet count is associated with septic shock and is a mortality predictor in patients hospitalised with CAP ${ }^{10,11}$. We could not find studies assessing platelet counts as predictor of mortality in children hospitalized with CAP. Since severe community acquired pneumonia is often associated with septicaemia which can lead to Disseminated Intravascular Coagulopathy leading to thrombocytopenia so these patients should be screened for thrombocytopenia at admission ${ }^{14}$.

Our study further revealed that SAM and abnormal sensorium, are independent predictors of mortality in patients hospitalised with CAP. Other pediatric studies ${ }^{4,15}$ have reported SAM as a mortality predictor of pneumonia. Tiewsoh K. et al also reported altered sensorium as a mortality predictor of pneumonia as in our study sensorium is found as an independent predictor of CAP ${ }^{3}$.

However more pediatric studies are required in this context and since the study was done at tertiary referral centre so the study may not be generalizable.

Inclusion and exclusion criteria were well defined and standard WHO classification and definitions were used which added to the strength of the study.

\section{Conclusion}

Controlling for SAM, abnormal sensorium and cyanosis, low platelet count increased risk of mortality in patients of CAP. Hence platelet count must be routinely estimated in cases of CAP.

\section{References}

1. WHO. Pneumonia Fact sheet. World Health Organization; 2015. Available from: http://www. who.int/mediacentre/factsheets/fs331/en/index.html (Accessed December 1, 2017).

2. WHO. Revised classification and treatment of childhood at health facilities. Evidence World Health Organization; 2014 Available at: http://apps.who.int/iris/bitstream/ 10665/137319/1/9789241507813 eng.pdf (Accessed December 1, 2017).

3. Tiewsoh K, Lodha R, Pandey RM, Broor S, Kalaivani M, Kabra SK. Factors determining the outcome of children hospitalized with severe pneumonia. BMC Pediatr. 2009;23(1):15 9.

4. Chisti MJ, Salam MA, Ashraf H, et al. Clinical risk factors of death from pneumonia in children with severe acute malnutrition in an urban critical care ward of bangladesh. PloS One. 2013;9(9):e73728 8.

5. Da Fonseca Lima EJ, Mello MJ, Lopes MI, Serra GH, Lima DE, Correia JB. Risk factors for community-acquired pneumonia in children under five years of age in the post-pn eumococcal conjugate vaccine era in Brazil: a case control study. BMC Pediatr. 2016;22(1):157 16

6. Agrawal S, Sachdev A, Gupta D, Chugh K. Platelet counts and outcome in the pediatric intensive care unit. IJCCM. 2008;12(3):102.

7. Yilmaz S, Yildizdas D, Acipayam C, et al. The effect of thrombocytopaenia on outcome in critically ill children. Crit Care Shock. 2013;16(2):48-57.

8. Kaur A, Sethi GK, Goyal RK, et al. Thrombocytopenia in Paediatric ICU: incidence, transfusion requirement and role as prognostic Indicator. $J$ Clin Diagn Res. 2015;9(12):SC05

9. Venkata C, Kashyap R, Farmer JC, Afessa B. Thrombocytopenia in adult patients with sepsis: incidence, risk factors, and its association with clinical outcome. J Intensiove 
Care. 2013;30(1):9 1.

10. Mirsaeidi M, Peyrani P, Aliberti S, et al. Thrombocytopenia and thrombocytosis at time of hospitalization predict mortality in patients with community-acquired pneumonia. Chest J. 2010;137(2):416-420.

11. Montull B, Menéndez R, Torres A, et al. Predictors of severe sepsis among patients hospitalized for community-acquired pneumonia. PLoS One. 2016;4(January, 1): $\mathrm{e} 0145929$.

12. WHO. Child Growth Standards World healthorganization; 2006. Available from: http://www.who.int/childgrowth/standards/Technical_report.pdf (Accessed December 1 2017).
13. WHO. Haemoglobin concentrations for the diagnosis of anaemia and assessment of severity. World Health Organization; 2011. Available from: http://www.who.int/ vmnis/indicators/haemoglobin/en/ (Accessed December 1 2017).

14. Semeraro N, Ammollo CT, Semeraro F, Colucci M. Sepsis-associated disseminated intravascular coagulation and thromboembolic disease. Mediterr J Hematol Infect Dis. 2010;2(3).

15. Lupisan SP, Ruutu P, Erma Abucejo-Ladesma P, et al. Predictors of death from severe pneumonia among children 2-59 months old hospitalized in Bohol, Philippines: implications for referral criteria at a first-level health facility. Trop Med Int Health. 2007;12(8):962-971 1. 